\title{
Combining Novel Probes and High Resolution Imaging to Dissect Mitochondrial Function in Living Systems
}

\author{
Karina Pena ${ }^{1}$, Mads Larsen ${ }^{1}$, Mike Calderon ${ }^{1}$, Michael Tsang ${ }^{2}$, Simon C. Watkins ${ }^{1}$, Marcel P. Bruchez ${ }^{3}$, \\ and Claudette M. St. Croix ${ }^{1}$ \\ 1. Center for Biologic Imaging, Department of Cell Biology \\ 2. Department of Developmental Biology University of Pittsburgh School of Medicine, Pittsburgh, USA. \\ ${ }^{3 .}$ Departments of Biological Sciences and Chemistry, Carnegie Mellon University, Pittsburgh, USA.
}

Mitochondria regulate energy production, $\mathrm{Ca}^{2+}$ signaling, reactive oxygen species (ROS) production and apoptosis in response to the physiological demands of the cell. They are extremely dynamic organelles that fragment and fuse continuously, and their morphology (fragmented vs. extensive fused networks) strongly influences function [1]. While microscopic methods have become increasingly important as fundamental tools to assess mitochondrial dynamics and function in living cells and/or organisms, in all instances, the ability to obtain temporal information at high resolution is critical to the success of these studies. In this paper, we describe the combined use of novel genetically targeted probes and high resolution optical imaging technologies to explore mitochondrial metabolism, ROS generation and function/dysfunction in the context of the living zebrafish (Danio rerio).

Super resolution methods circumvent the diffraction limit ( $200 \mathrm{~nm}$ in $\mathrm{xy})$ of conventional fluorescence imaging to provide spatial resolutions in the range of 10-100 nm. STED (STimulated Emission Depletion) microscopy, developed by Stefan Hell [2] is essentially a point scanning approach based around confocal technology, however, emission from the Gaussian spot used to excite the fluorophore in confocal microscopy is restricted to a smaller focal volume by a longer wavelength depletion source. This depletion source is shaped into a "donut" which quenches the fluorescence at the periphery of the excitation spot by stimulated emission. The advantages of using STED microscopy to study mitochondrial morphology/dynamics include the ability maintain accurate z-resolution at depth leading to accurate reproducible measurements mitochondrial size, fragmentation and volume.

The dye technology consists of single chain antibodies (Fluorogen Activating Peptides-FAPs) that bind to and activate fluorescence of nonfluorescent molecules [3]. These single-chain antibodies can be expressed as fusion constructs with cellular proteins, and the expressed proteins are labeled by addition of the complementary dye molecules with specific properties. This FAP-fluorogen technology, using triarylmethane (MG-based) fluorogens is suitable for both super-resolution microscopy and localization microscopy for cell surface and intracellular/cytosolic targets [4,5]. In Figure 1, we extend these findings to demonstrate the feasibility of using time-lapse STED imaging of mitochondrially targeted FAP in living HeLa cells with sufficient temporal and spatial resolution to track subtle changes in mitochondrial dynamics without appreciable photo-toxicity. We have also been successful in using these approaches to look at mitochondrial function in the context of the intact living organism. The transparency of the zebrafish (Danio rerio) embryo and its small size, make it an especially attractive tool for in vivo imaging. Figure 2 shows live STED microscopy of a within the epithelium of a 2 day post fertilization (dpf) transgenic zebrafish embryo expressing the FAP localized to mitochondria.

Lastly, we are testing a series of tandem dyes, consisting of a physiological indicator as a donor and an MG-based fluorogen as acceptor, that act as ratiometric physiological indicators for ROS. These sensors 
can bind the same FAP, and provide information about mitochondrial superoxide production in these cell and animal based model systems.

\section{References:}

[1] da Silva, A.F. et al, Cell Mol Life Sci 71(12) (2014), p. 2313.

[2] Klar, T.A., E. Engel, and S.W. Hell, Phys Rev E Stat Nonlin Soft Matter Phys 64(6.2) (2001), p. 066613.

[3] Szent-Gyorgyi C et al, Nat Biotechnol. 26(2) (2008), p. 235.

[4] Fitzpatrick JA et al, Bioconjug Chem. 20(10) (2009), p. 1843.

[5] Yan Q et al, Chemphyschem. 15(4) (2014), p. 687.
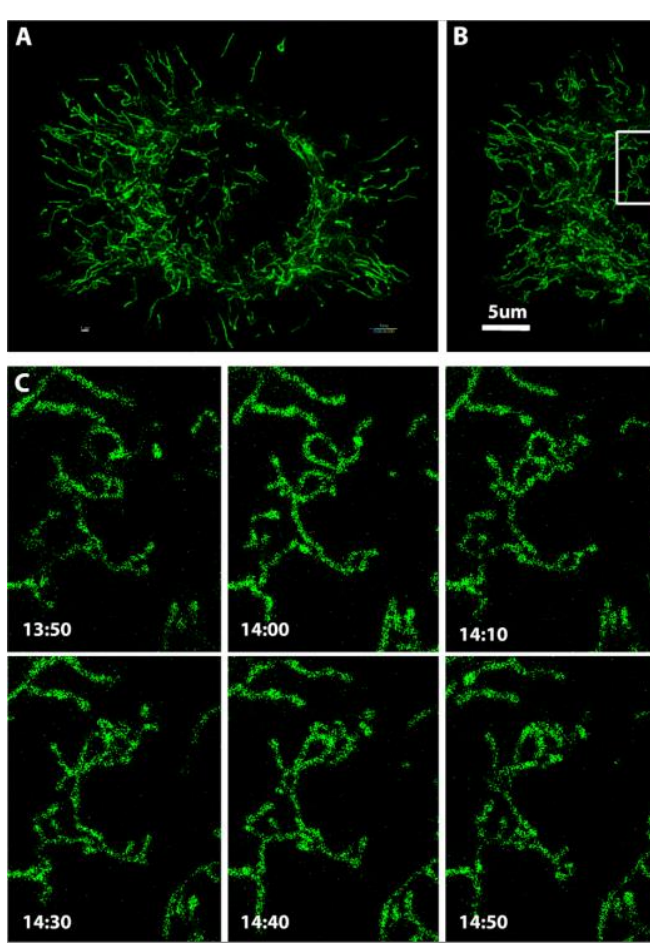

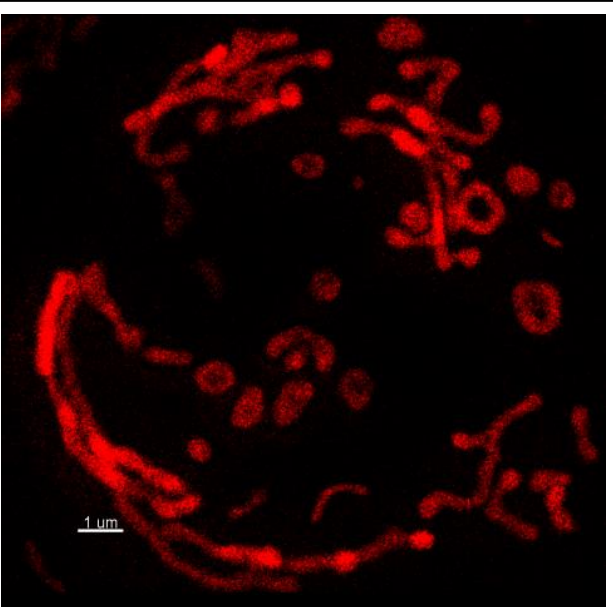

Figure 2. STED imaging of FAP localized to mitochondria within the epithelium of a living $2 \mathrm{dpf}$ zebrafish embryo. This image is taken from a series and is a snap shot after 15 minutes of continuous imaging. 